\title{
A GENERALIZATION OF STUDY'S THEOREM ON CONVEX MAPS
}

\author{
ROGER W. BARNARD
}

\begin{abstract}
Study proved that disks of radius $r, 0<r<1$, get mapped onto convex (starlike) sets under all convex (starlike) maps of the unit disk $U$.

Pommerenke and Heins gave a geometric characterization of the subsets of $U$ that get mapped onto convex sets under all convex maps. In this note we give an analytical characterization of the subsets of $U$ that get mapped onto $\alpha$-starlike domains under all $\alpha$-starlike maps of $U$ for all $\alpha>0$ noting that 0 -starlikeness equals starlikeness and 1-starlikeness equals convexity.
\end{abstract}

Introduction. For any number $r$, let $U_{r}=\{z:|z|<r\}$ and $U=U_{1}$. Let $S$ denote the collection of functions $f(z)=z+\ldots$ that are regular and univalent in $U$. We let $S^{*}=\{f: f \in S$ and $f(U)$ is starlike $\}$. Whenever we say a domain is starlike we mean that it is starlike with respect to the origin. Let $K=\{f: f \in S$ and $f(U)$ is convex $\}$. The requirement that $f^{\prime}(0)=1$ is not essential to the arguments in this paper. A subclass of $S$ defined by Mocanu in [6] gives a continuous parametrization between the starlike functions and the convex functions. For any fixed $\alpha, \alpha \geqslant 0$, this subclass is given by

$$
\begin{aligned}
S^{*}(\alpha)=\{f: & f(z) f^{\prime}(z) / z \neq 0, \\
& \left.\operatorname{Re}\left\{\alpha\left[1+z f^{\prime \prime}(z) / f^{\prime}(z)\right]+(1-\alpha)\left[z f^{\prime}(z) / f(z)\right]\right\}>0\right\} .
\end{aligned}
$$

We shall call functions in $S^{*}(\alpha) \alpha$-starlike functions. A number of authors [7] have shown that $S^{*}(\alpha)$ is a subclass of $S^{*}$ for $\alpha \geqslant 0$. We note that $S^{*}(0)=$ $S^{*}$ and $S^{*}(1)=K$. A domain $D$ will be called $\alpha$-starlike if and only if for every univalent function $f$ for which $f(U)=D$ we have $b(f)$ is in $S^{*}(\alpha)$ for some positive scalar $b$. We define for a fixed $\alpha, \alpha \geqslant 0$, the set $H_{S^{*}(\alpha)}=\{X \subset$ $U: f(X)$ is an $\alpha$-starlike domain under all $\alpha$-starlike maps $f$ of $U\}$. The cases $\alpha=0$ and $\alpha=1$ have been investigated by many authors.

In [9], Study showed that for any $r, 0<r \leqslant 1$, the disk $U_{r}$ is in $H_{S^{*}}$ and in $H_{K}$. In [1], [2] Alexandrov characterized those subsets of $U$ that are mapped onto convex domains as well as those that are mapped onto starlike domains under all regular univalent maps of $U$. Problem 6.20 in Hayman's book [3] asks the slightly more general question of characterizing members of $H_{K}$ but allows meromorphic convex maps of $U$. M. Reade proposed the problem of characterizing the sets of $H_{K}$ to $\mathrm{C}$. Pommerenke, who subsequently gave a

Received by the editors July 8, 1976.

AMS (MOS) subject classifications (1970). Primary 30A30, 30A32.

Key words and phrases. $\alpha$-starlikeness, Study's Theorem. 
solution to the problem in [8]. Later Heins [4], gave an alternate characterization of sets in $H_{K}$ including a topological representation for these sets. He continued this investigation in [5]. To discuss Pommerenke's and Heins's characterization we need to recall the notion of $D$-convexity (the name is due to Heins). Given two distinct points $a, b$ in $U$ there are exactly two circles $C_{0}$ and $C_{1}$ called oricycles which lie in the closure of $U$, pass through both $a$ and $b$, and have a tangent in common with the unit circle. We denote by $D(a, b)$ the intersection of the disks determined by $C_{0}$ and $C_{1}$. A subset $X$ of $U$ is said to be $D$-convex if $D(a, b)$ is contained in $X$ whenever $a$ and $b$ are distinct points of $X$. Pommerenke and Heins proved that $X$ is in $H_{K}$ if and only if $X$ is $D$-convex. In this paper we characterize the sets in $H_{S^{*}(\alpha)}$ for any fixed $\alpha \geqslant 0$, i.e. we characterize the subsets of $U$ that get mapped onto $\alpha$-starlike domains under all $\alpha$-starlike maps of $U$. Although this characterization does not have the particularly elegant geometric formulation that Heins's work has, this seems to be in the nature of things with respect to the more general class $S^{*}(\alpha)$. However, for the case $\alpha=1$ (our) Theorem 2 does give an analytical characterization of the sets described geometrically by Pommerenke and Heins.

REMARK. The author has recently been informed that for the starlike case of $\alpha=0$ a very interesting characterization of the sets in $H_{S^{*}}$, using a generalization of Heins's concept of $D$-convexity, has just been obtained by Peter Fenton.

Characterization of $H_{S^{*}}$. We first consider the simpler case when $\alpha=0$. Let $X$ be a subset of $U$. Since the identity map is in $S^{*}$ and since $X$ is starlike with respect to the origin we may assume $X$ is a starlike set containing the origin. The Riemann Mapping Theorem assures the existence of an univalent analytic function $w=w(z)$ on $U$ such that $w(U)=X, w(0)=0$ and $w^{\prime}(0)>$ 0 . Throughout this paper we will be using the principal branch of the log to define the 'arg' function. With this notation we shall characterize the members of $H_{S^{*}}$ in the following theorem.

THEOREM 1. Let $X$ be a subset of $U$ with the Riemann map $w, w(U)=X$, $w(0)=0$ and $w^{\prime}(0)>0$. Then $X$ is in $H_{S^{*}}$ if and only if

$$
\left|\arg \frac{z w^{\prime}(z)}{w(z)}\right| \leqslant \cos ^{-1} \frac{2|w(z)|}{1+|w(z)|^{2}}, \quad z \in U
$$

Proof. We first show that (1) implies $X$ is in $H_{S^{*}}$. We must show for any $f$ in $S^{*}$ that $f(X)$ is starlike. If we define $g$ on $U$ by $g(z)=f[w(z)]$, where $w(z)$ is the above Riemann map, then we only have to show that $g$ is in $S^{*}$. Since $f$ is in $S^{*}$ there exists a Schwarz function $\phi$, analytic on $U, \phi(0)=0$ and $|\phi(w)| \leqslant|w|$, such that $w f^{\prime}(w) / f(w)=(1+\phi(w)) /(1-\phi(w))$. Thus 


$$
\left|\arg \frac{z g^{\prime}(z)}{g(z)}\right|=\left|\arg \frac{z f^{\prime}(w) w^{\prime}(z)}{f(w)}\right| \leqslant\left|\arg \frac{z w^{\prime}}{w}\right|+\left|\arg \frac{1+\phi(w)}{1-\phi(w)}\right| .
$$

Since $|\phi(w)| \leqslant|w|<1,(1+\phi) /(1-\phi)$ is contained in the disk of radius $2|\phi| /\left(1-|\phi|^{2}\right)$ with center $\left(1+|\phi|^{2}\right) /\left(1-|\phi|^{2}\right)$. Thus the right-hand side of (2) is less than or equal to

$$
\left|\arg \frac{z w^{\prime}}{w}\right|+\sin ^{-1}\left(\frac{2|\phi|}{1+|\phi|^{2}}\right) \leqslant\left|\arg \frac{z w^{\prime}}{w}\right|+\sin ^{-1}\left(\frac{2|w|}{1+|w|^{2}}\right)
$$

which is less than or equal to $\pi / 2$ whenever

$$
\left|\arg \frac{z w^{\prime}(z)}{w(z)}\right| \leqslant \frac{\pi}{2}-\sin ^{-1}\left(\frac{2|w|}{1+|w|^{2}}\right)=\cos ^{-1}\left(\frac{2|w|}{1+|w|^{2}}\right) .
$$

Thus if (1) holds then $g(U)=f(X)$ is starlike for each $f \in S^{*}$.

For the converse assume there exists a point $w_{0}$ in $X$ such that $w_{0}=w\left(z_{0}\right)$ where $\left|\arg \left(z_{0} w^{\prime}\left(z_{0}\right) / w\left(z_{0}\right)\right)\right|>\cos ^{-1} 2\left|w\left(z_{0}\right)\right| /\left(1+\left|w\left(z_{0}\right)\right|^{2}\right)$. Since $X$ is at least starlike, then by a rotation and or reflection of $X$ we can assume $w_{0}>0$ and

$$
0 \leqslant \arg \left[z_{0} w^{\prime}\left(z_{0}\right) / w\left(z_{0}\right)\right]<\pi / 2 .
$$

By choosing $f_{0}(z)=z(1-i z)^{-2}$ in $S^{*}$ we have

$$
\begin{aligned}
\left|\arg \frac{z_{0} g^{\prime}\left(z_{0}\right)}{g\left(z_{0}\right)}\right| & =\arg \frac{z_{0} w^{\prime}\left(z_{0}\right)}{w\left(z_{0}\right)}+\arg \frac{1+i w_{0}}{1-i w_{0}} \\
& >\cos ^{-1}\left(\frac{2 w_{0}}{1+w_{0}^{2}}\right)+\sin ^{-1}\left(\frac{2 w_{0}}{1+w_{0}^{2}}\right)=\frac{\pi}{2} .
\end{aligned}
$$

Thus, it follows that $b(f)$ is not in $S^{*}$ for any scalars. This completes the proof of Theorem 1 .

Characterization of $H_{S^{*}(\alpha)}$. In order to do the more general case of $\alpha \geqslant 0$, a bound is needed for the argument of a sum rather than the argument of a product as in (2). Thus a different approach is required. We present some preliminary notation. Given a function $h$, analytic on $U, h(0)=0$, $h(z) h^{\prime}(z) / z \neq 0$, we define for a given $\alpha \geqslant 0$ and $r, 0<r<1$,

$$
J_{h}(\theta)=(1-\alpha) \arg h\left(r e^{i \theta}\right)+\alpha\left[\arg \left(r e^{i \theta}\right) h^{\prime}\left(r e^{i \theta}\right)\right] .
$$

Since for each $\alpha \geqslant 0 f(z)=z$ is in $S^{*}(\alpha)$, we may assume that the set $X$ in $H_{S^{*}(\alpha)}$ is at least starlike and contains the origin. Let $w$ be the Riemann map such that $w(U)=X, w(0)=0$, and $w^{\prime}(0)>0$. For any $r, 0<r<1$, let $w_{0}=w\left(r e^{i \theta_{0}}\right)$ and $w_{1}=w\left(r e^{i \theta_{1}}\right)$ be two distinct points in $X$. The starlikeness of $X$ assures that $w(z) w^{\prime}(z) / z \neq 0$ for $z$ in $U$. We may assume $0<$ $\arg \left(w_{1} / w_{0}\right) \leqslant \pi$ since $X$ is starlike $r<1$, and

$$
\operatorname{Re}\left\{z w^{\prime}(z) / w(z)\right\}=\frac{\partial}{\partial \theta}\left[\arg w\left(r e^{i \theta}\right)\right]>0
$$


so that $\arg w_{0} \neq \arg w_{1}$. Thus $0 \leqslant \theta_{0}<\theta_{1}<2 \pi$. Let the two uniquely determined oricycles passing through $w_{0}$ and $w_{1}$ be denoted by $C_{0}$ and $C_{1}$, with centers $\eta_{0}, \eta_{1}$ and the common points of tangency to the unit circle by $\zeta_{0}$, $\zeta_{1}$, respectively. One of the common points of tangency say $\zeta_{0}$ is such that

$$
0<\arg \frac{w_{1}-\zeta_{0}}{w_{0}-\zeta_{0}}<\pi
$$

From a geometric property of a circle we have that

$$
2 \arg \frac{w_{1}-\zeta_{0}}{w_{0}-\zeta_{0}}=\arg \frac{w_{1}-\eta_{0}}{w_{0}-\eta_{0}}
$$

when $0<\arg \left[w_{1} / w_{0}\right] \leqslant \pi$.

With this notation we will characterize the members of $H_{S^{*}(\alpha)}$ in the following theorem.

THEOREM 2. Given any nonnegative $\alpha, X$ is in $H_{S^{*}(\alpha)}$ if and only if for each $r$, $0<r<1$, and $0 \leqslant \theta_{0}<\theta_{1}<2 \pi$ such that

$$
0<\arg \left[w\left(r e^{i \theta_{1}}\right) / w\left(r e^{i \theta_{0}}\right)\right] \leqslant \pi
$$

we have

$$
J_{w}\left(\theta_{1}\right)-J_{w}\left(\theta_{0}\right) \geqslant \arg \frac{w\left(r e^{i \theta_{1}}\right)-\eta_{0}}{w\left(r e^{i \theta_{0}}\right)-\eta_{0}}
$$

with $J_{w}(\theta)$ defined by (3).

Proof. We shall first prove the sufficiency of condition (5). Let $f$ be in $S^{*}(\alpha)$ and define $g(z)=f[w(z)]$. Then we need to prove that $g(U)$ is $\alpha$-starlike, i.e., we need to show that

$$
\operatorname{Re}\left\{\alpha\left[1+\frac{z g^{\prime \prime}(z)}{g^{\prime}(z)}\right]+(1-\alpha)\left[\frac{z g^{\prime}(z)}{g(z)}\right]\right\}=\frac{\partial J_{g}(\theta)}{\partial \theta}
$$

is nonnegative in $|z| \leqslant r<1$. Since $\partial J_{g}(\theta) / \partial \theta$ is harmonic it suffices by the minimum principle to consider only $|z|=r$.

Using the definition of $g$ we obtain

$$
\frac{z g^{\prime}(z)}{g(z)}=\frac{w f^{\prime}(w)}{f(w)} \cdot \frac{z w^{\prime}(z)}{w(z)}
$$

and

$$
1+\frac{z g^{\prime \prime}(z)}{g^{\prime}(z)}=\frac{z w^{\prime}(z)}{w(z)} \cdot \frac{w f^{\prime \prime}(w)}{f^{\prime}(w)}+1+\frac{z w^{\prime \prime}(z)}{w^{\prime}(z)}
$$

Thus 


$$
\begin{aligned}
& \frac{\partial J_{g}(\theta)}{\partial \theta}=\operatorname{Re}\left\{\alpha\left[1+\frac{z w^{\prime \prime}(z)}{w^{\prime}(z)}+\frac{z w^{\prime}(z)}{w(z)} \cdot \frac{w f^{\prime \prime}(w)}{f^{\prime}(w)}\right]\right. \\
&\left.+(1-\alpha)\left[\frac{z w^{\prime}(z)}{w(z)} \cdot \frac{w f^{\prime}(w)}{f(w)}\right]\right\} \\
&=\operatorname{Re}\left\{\alpha\left[1+\frac{z w^{\prime \prime}(z)}{w^{\prime}(z)}+\frac{z w^{\prime}(z)}{w(z)}\left[\alpha \frac{w f^{\prime \prime}(w)}{f^{\prime}(w)}\right]+(1-\alpha) \frac{w f^{\prime}(w)}{f(w)}\right]\right\} \\
&\left.=\operatorname{Re}\left\{\alpha\left[1+\frac{z w^{\prime \prime}(z)}{w^{\prime}(z)}\right]-\alpha \frac{z w^{\prime}(z)}{w(z)}\right]+\frac{z w^{\prime}(z)}{w(z)}\left[\alpha\left(1+\frac{w f^{\prime \prime}(w)}{f^{\prime}(w)}\right)+(1-\alpha) \frac{w f^{\prime}(w)}{f(w)}\right]\right\} \\
&=\operatorname{Re}\left\{\alpha\left[1+\frac{z w^{\prime \prime}(z)}{w^{\prime}(z)}\right]-\alpha \frac{z w^{\prime}(z)}{w(z)}+\frac{z w^{\prime}(z)}{w(z)}\left[\frac{1}{2 \pi} \int_{0}^{2 \pi} \frac{e^{i t}+w}{e^{i t}-w} d u(t)\right]\right\},(7)
\end{aligned}
$$

where the existence in (7) of a nonnegative, nondecreasing measure $u$ on $[0,2 \pi]$ with $u(2 \pi)-u(0)=2 \pi$ is guaranteed since $f$ is in $S^{*}(\alpha)$.

Letting $z=r e^{i \theta}$ we see that the nonnegativeness of (7) is equivalent to the nonnegativeness of the following quantity for all $\theta_{0}, \theta_{1}, 0 \leqslant \theta_{0}<\theta_{1}<2 \pi$ and $\theta_{1}-\theta_{0}$ sufficiently small

$$
\begin{aligned}
\int_{\theta_{0}}^{\theta_{1}} \operatorname{Re}\left\{\alpha\left(1+\frac{z w^{\prime \prime}(z)}{w^{\prime}(z)}\right)-\alpha \frac{z w^{\prime}(z)}{w(z)}+\frac{z w^{\prime}(z)}{w(z)}\left[\frac{1}{2 \pi} \int_{0}^{2 \pi} \frac{e^{i t}+w}{e^{i t}-w} d u(t)\right]\right\} d \theta \\
=\int_{\theta_{0}}^{\theta_{1}} \operatorname{Re}\left\{\alpha\left[1+\frac{z w^{\prime \prime}(z)}{w^{\prime}(z)}\right]+(1-\alpha) \frac{z w^{\prime}(z)}{w(z)}\right\} d \theta \\
+\operatorname{Re}\left\{\int_{\theta_{0}}^{\theta_{1}} \frac{z w^{\prime}(z)}{e^{i t}-w} d u(t) d \theta\right\} \\
=\int_{\theta_{0}}^{\theta_{1}}\left[\frac{\partial J_{w}(\theta)}{\partial \theta}\right] d \theta+\operatorname{Re}\left\{\frac{1}{\pi} \int_{0}^{2 \pi}\left[\int_{\theta_{0}}^{\theta_{1}} \frac{z w^{\prime}(z)}{e^{i t}-w} d \theta\right] d u(t)\right\}
\end{aligned}
$$

The interchanging of the order of integration follows from the continuity in $\theta$ and $t$ of the integrand of the second term in (8). Since $z=r e^{i \theta}$ it follows for a fixed $r$ that $d z=i r e^{i \theta} d \theta$. This fact and remembering that $w(z)$ is one-to-one allows us to rewrite (9) as 


$$
\begin{gathered}
J_{w}\left(\theta_{1}\right)-J_{w}\left(\theta_{0}\right)-\frac{1}{2 \pi} \int_{0}^{2 \pi} 2 \operatorname{Im}\left\{\int_{w_{0}}^{w_{1}} d\left[\log \left(e^{i t}-w\right)\right]\right\} d u(t) \\
=J_{w}\left(\theta_{1}\right)-J_{w}\left(\theta_{0}\right)-\frac{1}{2 \pi} \int_{0}^{2 \pi}\left[2 \arg \left(\frac{e^{i t}-w_{1}}{e^{i t}-w_{0}}\right)\right] d u(t) \\
\geqslant J_{w}\left(\theta_{1}\right)-J_{w}\left(\theta_{0}\right)-2 \max _{|\zeta|=1} \arg \frac{\zeta-w_{1}}{\zeta-w_{0}} .
\end{gathered}
$$

It follows that (7) is nonnegative whenever

$$
J_{w}\left(\theta_{1}\right)-J_{w}\left(\theta_{0}\right) \geqslant 2 \max _{|\zeta|=1} \arg \frac{\zeta-w_{1}}{\zeta-w_{0}} .
$$

We now prove that

$$
\max _{|\zeta|=1} \arg \frac{\zeta-w_{1}}{\zeta-w_{0}}=\arg \frac{\zeta_{0}-w_{1}}{\zeta_{0}-w_{0}}
$$

where $\zeta_{0}=e^{i \theta_{0}}$ is the point on the oricycle defined by (4). For an arbitrary point $\zeta$ on the unit circle, let $\beta=\arg \left[\left(\zeta-w_{1}\right) /\left(\zeta-w_{0}\right)\right]$. Because we are determining the max of $\arg \left[\left(\zeta-w_{1}\right) /\left(\zeta-w_{0}\right)\right]$ we need only consider the $\zeta$ 's in the half plane determined by the line through $w_{0}$ and $w_{1}$ that makes $\beta$ positive. We let $w_{\zeta}$ be the point of intersection of the oricycle $C_{0}$ and the line segment $\overline{\zeta w_{0}}$. Denote by $\gamma$ the angle $w_{1} w_{\zeta} w_{0}$. Then, since $\zeta$ is not inside $C_{0}$, it is clear that $\beta \leqslant \gamma$. From the simple geometric properties of a circle it follows that $\gamma$ equals the angle $w_{1} \zeta_{0} w_{0}$. Hence (13) follows. Since the central angle $w_{1} \eta_{0} w_{0}$ equals twice the angle $w_{1} \zeta_{0} w_{0}$ condition (5) implies that $g(U)$ is $\alpha$-starlike. Therefore $f(X)$ is $\alpha$-starlike for each $f$ in $S^{*}(\alpha)$.

To prove that (5) is a necessary condition for $X$ to be in $H_{S^{*}(\alpha)}$ assume there exists an $X$ in $H_{S^{*}(\alpha)}$ with the Riemann map $w^{*}$ and that $X$ contains two points $w_{0}^{*}=w^{*}\left(r e^{i \theta_{0}}\right), w_{1}^{*}=w^{*}\left(r e^{i \theta_{1}}\right), 0<\arg \left[w_{1}^{*} / w_{0}^{*}\right] \leqslant \pi, 0 \leqslant \theta_{1}<\theta_{2}<$ $2 \pi$ such that

$$
J_{w^{*}}\left(\theta_{1}\right)-J_{w^{*}}\left(\theta_{0}\right)<\arg \frac{\eta_{0}^{*}-w_{1}^{*}}{\eta_{0}^{*}-w_{0}^{*}}=2 \arg \frac{\zeta_{0}^{*}-w_{1}^{*}}{\zeta_{0}^{*}-w_{0}^{*}}
$$

with $\zeta_{0}^{*}, \eta_{0}^{*}$ defined as functions of $w_{1}^{*}$ and $w_{0}^{*}$ as before. Let $\zeta_{0}^{*}=e^{i \theta_{3}}$. Choose $u=u_{0}(t)$ such that

$$
u_{0}(t)= \begin{cases}0, & 0 \leqslant t \leqslant \theta_{3} \\ 2 \pi, & \theta_{3}<t \leqslant 2 \pi\end{cases}
$$

in

$$
\alpha\left[1+\frac{z f_{u_{0}}^{\prime \prime}(z)}{f_{u_{0}}^{\prime}(z)}\right]+(1-\alpha) \frac{z f_{u_{0}}^{\prime}(z)}{f_{u_{0}}(z)}=\frac{1}{2 \pi} \int_{0}^{2 \pi} \frac{e^{i t}+z}{e^{i t}-z} d u(t) .
$$

This defines $f_{u_{0}}$ where $f_{u_{0}}$ has the form 


$$
f_{u_{0}}(z)=\left[\frac{1}{\alpha} \int_{0}^{z} \zeta^{1 / \alpha-1}\left(1-\bar{\zeta}_{0}^{*} \zeta\right)^{-2 / \alpha} d \zeta\right]^{\alpha}
$$

This $f_{u_{0}}$, where the appropriate branches have been chosen in the above integral, is a rotation of the generalized Köebe function shown by many authors to be the extremal function for a number of problems over the class $S^{*}(\alpha)$. Replacing $u$ by $u_{0}$ in (10) gives equality between lines (10) and (11). Thus, for the function $g^{*}$ defined by $g^{*}(z)=f_{u_{0}}\left[w^{*}(z)\right]$ we obtain

$$
J_{g^{*}}\left(\theta_{1}\right)-J_{g^{*}}\left(\theta_{0}\right)=J_{w^{*}}\left(\theta_{1}\right)-J_{w^{*}}\left(\theta_{0}\right)-2 \arg \frac{\zeta_{0}^{*}-w_{1}^{*}}{\zeta_{0}^{*}-w_{0}^{*}}<0
$$

It follows that $g^{*}(z)$ maps $D$ onto $X$ and that $b(g)$ is not in $S^{*}(\alpha)$ for any positive scalar $b$. This completes the proof of Theorem 2 .

REMARK. When $\alpha=1$ condition (5) in Theorem 2 becomes

$$
\arg r e^{i \theta_{1} w^{\prime}}\left(r e^{i \theta_{1}}\right)-\arg r e^{i \theta_{0} w^{\prime}}\left(r e^{i \theta_{0}}\right) \geqslant \arg \frac{w\left(r e^{i \theta_{1}}\right)-\eta_{0}}{w\left(r e^{i \theta_{0}}\right)-\eta_{0}}
$$

To see that (15) gives an analytic description of the geometric criterion of Pommerenke-Heins is a long but straightforward argument based essentially on the observation that equality in (15) exactly characterizes an oricycle.

One may ask if there is a geometric characterization of the sets in $H_{S^{*}(1)}=H_{K}$. The answer is that such sets are made up of arbitrary intersections of sets having oricycles as boundaries. Since oricycles appear in Theorem 2 for any $\alpha$, one can ask for what $\alpha$ other than $\alpha=1$, can an oricycle form the boundary of a set in $H_{S^{*}(\alpha)}$. It follows from Theorem 2 by a lengthy continuity argument that the only value of $\alpha$ for which a set in $H_{S^{*}(\alpha)}$ has an oricycle as its boundary is $\alpha=1$. The author wishes to thank the referee for many helpful suggestions in this paper.

\section{REFERENCES}

1. I. A. Alexandrov, Conditions for convexity of the image region under mappings by functions regular and univalent in the unit circle, Izv. Vyš̌s. Učebn. Zaved. Matematika, 1958 no. 6 (7) 3-6. (Russian)

2. On the star-shaped character of the mappings of a domain by functions that are regular and univalent in the circle, Izv. Vysš. Učebn. Zaved. Mathematika 1959 no. 4 (11) 9-15. (Russian)

3. W. K. Hayman, Research problems in function theory, The Athlone Press [University of London], 1967. MR 36 \#359.

4. M. Heins, On a theorem of Study concerning conformal maps with convex images, Mathematical Essays Dedicated to A. J. Macintyre, pp. 171-176, Ohio Univ. Press, Athens, Ohio, 1970. MR 42 \#7878.

5. On a theorem of Study concerning conformal maps with convex images. II, Math. Scand. 32 (1973), 245-257. MR 49 \# 551.

6. P. T. Mocanu, Une propriété de convexité généralisée dans la théorie de la représentations ronforme, Mathematica (Cluj) 11 (34) (1969), 127-133. MR 42 \#7881. 
7. S. S. Miller, P. Mocanu and M. O. Reade, All $\alpha$-convex functions are starlike, Proc. Amer. Math. Soc. 37 (1973), 553-554. MR 48 \#2364.

8. C. Pommerenke, Images of convex domains under convex conformal mappings, Michigan Math. J. 9 (1962), 257. MR 26 \#6378.

9. E. Study, Vorlesungen über ausgewählte Gegenstä der Geometrie, Heft 2, Konforme Abbildung einfach zusammenhängender Bereiche, B. G. Teubner, Leipzig and Berlin, 1913.

Department of Mathematics, TeXas Tech University, Lubbock, TeXas 79409 